\title{
Trichoderma en la Argentina: Estado del arte
}

\author{
Natalia S. Amerio ${ }^{1,2}$; ; María L. Castrillo ${ }^{12} ;$ Gustavo A. Bich ${ }^{12,}$; Pedro D. Zapata ${ }^{1 / 2}$ \\ \& LAura L. Villalba ${ }^{1}$ \\ ${ }^{1}$ Instituto de Biotecnología Misiones "Dra. María Ebe Reca" - InBioMis, Universidad Nacional de Misiones. ${ }^{2}$ CONICET \\ (Consejo Nacional de Investigaciones Científicas y Técnicas).
}

\begin{abstract}
RESUMEN. Las metodologías que se aplican para manejar los sistemas agrícolas y agroforestales tienen efectos notables en la producción de alimentos y en la conservación de la biodiversidad. Las enfermedades que causan los hongos fitopatógenos son uno de los problemas críticos en la agricultura argentina. A partir de la necesidad de encontrar mecanismos que eleven la productividad del campo, en los últimos años se intensificó la búsqueda de estrategias de control de enfermedades agrícolas que sean alternativas al control químico, buscando, además, disminuir los riesgos ambientales y sanitarios derivados de éste. El hongo Trichoderma es uno de los agentes que más se usan en los programas de control biológico como reguladores de hongos fitopatógenos. Esta revisión tiene como objetivo realizar una compilación de toda la información disponible respecto al estado del arte de Trichoderma como controlador biológico en la Argentina, con el fin de referenciar diferentes instituciones del país que centralizan a este hongo en sus investigaciones. Cerca de 70 grupos de universidades e institutos de investigación describieron aproximadamente diez especies diferentes de Trichoderma con capacidad antagonista, se estudiaron alrededor de 35 cultivos y 40 patógenos. La especie de Trichoderma más utilizada como agente de control biológico es Trichoderma harzianum, seguida por Trichoderma koningii y Trichoderma viride. Actualmente, en el mercado argentino existen cerca de 14 bioproductos cuyo principio activo son cepas de Trichoderma. Las crecientes iniciativas de las instituciones nacionales sobre estos estudios son básicas para el desarrollo de tecnologías innovadoras y para lograr una agricultura más productiva y ambientalmente sostenible en el país.
\end{abstract}

[Palabras clave: agricultura, control biológico, cultivos agronómicos, fitopatógenos]

\begin{abstract}
Aвstract. Trichoderma in Argentina: State of art. The methodologies applied in agricultural and agroforestry systems management have great impacts on food production and biodiversity conservation. Diseases caused by phytopathogenic fungi are one of the major problems in the agriculture of Argentina. The need to find mechanisms that increase the productivity of agricultural and agroforestry systems has driven the search for less risky and more environmentally friendly control strategies of agricultural diseases. Trichoderma fungus is one of the most commonly used agents in biological control programs as a phytopathogenic fungi regulator. This review aims to compile all the available information regarding the state of art of Trichoderma in Argentina as a biological control agent, in order to reference different institutions of the country that focus their research on this fungus. Nearly 70 groups, representing universities and research institutes, have described approximately ten different species of Trichoderma with antagonistic capacity associated to 35 crops and 40 pathogens. The most used Trichoderma species as a biological control agent is Trichoderma harzianum, followed by Trichoderma koningii and Trichderma viride. Currently, there are around 14 bioproducts in the argentine market whose active principles are strains of Trichoderma. The growing initiatives of the national institutions on these studies are crucial for the development of innovative technologies to achieve a more productive and environmentally sustainable agriculture for the country.
\end{abstract}

[Keywords: agriculture, agronomic crops, biological control, phytopathogen]

\section{INTRODUCCIÓN}

Las enfermedades de las plantas constituyen una de las causas fundamentales de la destrucción de los recursos naturales en la agricultura (Benítez et al. 2004). Las enfermedades causadas por fitopatógenos habitantes del suelo son uno de los problemas fitosanitarios más importantes. Esos fitopatógenos incluyen especies de hongos, bacterias y nematodos que pueden destruir las semillas u otros órganos de propagación, causar daño en plántulas, pudrición y destrucción de raíces o marchitez debido a

Editor asociado: Martín Nuñez deterioros en el sistema vascular (Bettiol 2006). Las enfermedades causadas por fitopatógenos fúngicos suelen ser una limitante en la producción de cualquier cultivo, por lo que su control es un factor clave a considerar (Fravel 2005). La forma de control tradicional de las enfermedades fúngicas en cultivos agrícolas es aplicar productos químicos, pero debido a su composición pueden resultar tóxicos e inespecíficos dañando la flora del suelo (Vinale et al. 2008). Por lo tanto, un reto importante para los investigadores y la industria es el desarrollo de nuevos agentes de control biológico y de bioproductos de

Recibido: 22 de Abril de 2019

Aceptado: 13 de Diciembre de 2019 
calidad que estén disponibles en el mercado. Entre las situaciones a resolverse destacan el desarrollo de metodologías para la producción a gran escala de agentes de control biológico y su transferencia al sector privado, el desarrollo de formulaciones que promuevan la facilidad de uso y conservación de los productos biológicos, el desarrollo de métodos para la evaluación de la calidad y la estabilidad de los productos basados en agentes de control biológico y el desarrollo de estrategias para concientizar a la sociedad sobre la importancia de la transición de una agricultura tradicional hacia la agroecología (Bettiol and Ghini 2014). En relación con los agricultores, es necesario poner a disposición bioproductos con precios ventajosos, diseñar campañas educativas destinadas a la transferencia de información sobre las particularidades y cuidados de estos insumos biológicos, efectuar entrenamientos específicos a productores y técnicos para que los manipulen y apliquen correctamente. En relación con la difusión de uso de los bioproductos es relevante promover adecuadamente el agente de control biológico, diseñar campañas dirigidas a agricultores, técnicos y consumidores sobre las ventajas de éstos, incluir en los currículos de las carreras de Ingeniería Agronómica y Forestal disciplinas específicas sobre control biológico, y entrenar a vendedores y agentes de asistencia técnica sobre los agentes de control biológico y sus especificidades (Rivera and Wright 2014). El objetivo de este trabajo fue realizar una compilación de toda la información disponible respecto al estado del arte de Trichoderma en la Argentina como agente de control biológico de distintos hongos fitopatógenos.

\section{Control de plagas y enfermedades}

A lo largo de la historia, con el propósito de reducir las pérdidas ocasionadas por las plagas y enfermedades de los cultivos se han desarrolladodiversosmétodosimplementando numerosos programas de control; el control químico ha sido el método tradicional de mayor difusión para combatirlos. Se trata del uso de pesticidas químicos, es decir, de aplicar de manera directa productos sintéticos de reconocida actividad biológica sobre plagas o patógenos (Bettiol 2006).

Según datos de la Organización de las Naciones Unidas para la Alimentación y la Agricultura (FAO) en el 2015, respecto de la utilización de herbicidas por superficie arable por país, la Argentina está en el segundo lugar de mayor utilización de herbicidas por hectárea en sus sistemas productivos. Según datos brindados por la Cámara de la Industria Argentina de Fertilizantes y Agroquímicos (CIAFA), en 2017, en la Argentina se consumieron 3.8 millones de toneladas de agroquímicos. La producción de soja acaparó casi el 62\% del total de los productos aplicados, el 10\% fue al cultivo de maíz y el $28 \%$ a otros cultivos como trigo, cebada, vid, caña, frutas de pepita y carozo. En un ranking de los diez productos más usados en la Argentina durante los últimos años, aparecen glifosato, 2,4-D, atrazina, diclosulam, cletodim, azoxistrobina, cyproconazol, rynaxypyr, haloxifop, clorpirifos y flubendiamida (Rivera and Wright 2014). El glifosato es el agroquímico más usado; concentra el 64\% del total de las ventas. Durante la última década, además del incremento en la superficie tratada con glifosato también aumentó el principio activo que se usa para elaborarlo. La Argentina se convirtió en el país con mayor consumo de glifosato del mundo, ya que se utilizan $4.3 \mathrm{~L}$ de este agroquímico por persona, mientras que en los Estados Unidos de América se usan $0.42 \mathrm{~L}$ por habitante; por otra parte, en Francia, el herbicida está prohibido por las consecuencias que genera en la salud de las personas (Vázquez et al. 2017).

Se sugiere que el mecanismo de acción del glifosato no se limita sólo a las plantas, sino que puede alterar la estructura del ADN en otros tipos de células como son las de los mamíferos (Walsh et al. 2000; Paganelli et al. 2010). En las zonas fumigadas, 6 de cada 100 niños nacen con problemas neurológicos, cardíacos y síndrome de down. El triple de niños nace con malformaciones, muchos de ellos mueren, otros quedan con discapacidades (Monroy et al. 2005).

Por otra parte, el uso inadecuado y continuo de estos pesticidas sintéticos acarrea altos costos ambientales. En un estudio reciente llevado a cabo por diversas instituciones de la Argentina detectaron la presencia alarmante de agroquímicos y metales pesados en la cuenca del Río Paraná, uno de los más influyente del país por su caudal, por la extensión de su área tributaria y por la longitud de su curso (Etchegoyen et al. 2017). En los monitoreos ambientales realizados en la ciudad de La Plata, Argentina, por investigadores del Consejo Nacional de Investigaciones Científicas y Técnicas (CONICET) en enero de 2017, se ratificó la presencia de agroquímicos tanto en el agua como en los sedimentos del río de La Plata. Los especialistas manifestaron 
que las muestras obtenidas presentaban efectos letales (organismos muertos) y subletales (alteración del desarrollo y la reproducción) (Etchegoyen et al. 2017). Cabe mencionar también que la actividad de estos agroquímicos afecta directa e indirectamente a otros organismos no-blanco por su capacidad de bioacumulación, su inespecificidad y su persistencia en el ambiente (tanto en el suelo como en aire y agua), alterando de esta manera diversas interacciones ecológicas (Pimentel et al. 1991). Dicho fenómeno fue demostrado por científicos del Instituto Nacional de Limnología de la Provincia de Santa Fe, Argentina, quienes observaron que el glifosato, tanto en su formulación comercial como el principio activo puro, produce efectos letales, cambios en el consumo de oxígeno y alteración en el crecimiento y comportamiento del cangrejo Trichodactylus borellianus (Decapoda: Trichodactylidae) (Nobili 1896), especie clave que sostiene la relaciones tróficas de los ambientes lénticos donde se desarrollan (Montagna and Collins 2004). Además, el uso intensivo de agroquímicos tiende a generar resistencia en las poblaciones biológicas y un rápido crecimiento de plagas secundarias, siendo a largo plazo mayores los riesgos que los beneficios de su uso (Vinale et al. 2008). Debido a la toma de conciencia de dichos problemas y a las exigencias internacionales sobre las restricciones en el uso de productos químicos en los alimentos de exportación, se intensificó la búsqueda de estrategias de control de enfermedades agrícolas que sean alternativas al control químico. Estas estrategias deben ser eficientes y no dañar el ambiente, con el fin de reducir el riesgo ambiental y sanitario sin arriesgar la salud humana. Esto constituye, hoy en día, un gran reto para la agricultura y su desarrollo (Altieri and Nicholls 2000).

El control biológico se puede definir como "la acción de los enemigos naturales (i.e., depredadores, parasitoides y patógenos) para mantener la población de otro organismo a densidades inferiores de las que tendría en su ausencia" (DeBach 1964). El control biológico es una de las herramientas del manejo integrado de plagas (MIP) que adquirió relevancia por la preocupación de la preservación del ambiente y la inocuidad alimentaria. El MIP es un conjunto de técnicas de control que son eficaces desde el punto de vista biológico, ecológico y económico, que tienen como objetivo emplear elementos naturales para regular poblaciones de plagas o patógenos por debajo del nivel de daño que sería económicamente aceptable (Kogan 1998). Este concepto brinda una respuesta a muchos problemas de la agricultura, como aumentar la producción con los recursos existentes, evitar la aparición del fenómeno de la resistencia de los patógenos a los fungicidas, disminuir la polución y los riesgos del manipuleo de plaguicidas y posibilitar mantener una agricultura continua (Kogan 1998). Las investigaciones sobre control biológico de patógenos de plantas se vienen desarrollando desde la década del '30; sin embargo, disminuyeron después del descubrimiento de moléculas de plaguicidas sintéticas durante de la Segunda Guerra Mundial. Tiempo después se renovó el interés en el manejo biológico de plagas debido a la demostración por ambientalistas y ecólogos de que la aplicación amplia y repetida de estas moléculas sintéticas podría ser ecológicamente dañina (Cook and Baker 1983). El primer éxito en el campo de control biológico de fitopatógenos (objetivo Sclerotium rolfsii, Atheliales: Atheliaceae) (Saccardo 1911) se registró utilizando Trichoderma durante los años '70 (Wells et al. 1972). A partir de entonces, a pesar de los problemas que continúan enfrentando los ecólogos para la aplicación exitosa de programas de control biológico, el estudio y la aplicación de microorganismos o sus productos (principalmente enzimas) como agentes de control biológico para ciertas enfermedades fue en ascenso. El control biológico se considera un método alternativo, sostenible y amigable con el ambiente (DeBach 1964). Las especies de Trichoderma son los agentes de control biológico más ampliamente utilizados en el manejo de enfermedades fúngicas de cultivos (Mukherjee et al. 2013).

\section{El rol del género Trichoderma en la agricultura}

Los hongos pertenecientes al género Trichoderma se caracterizan por ser microorganismos saprófitos, que sobreviven en suelos con diferentes tipos y cantidades de materia orgánica, a diferente temperatura, humedad, pH y contenido de nutrientes. Esta adaptabilidad permite que se encuentren ampliamente distribuidos y que sean muy benéficos para la agricultura (Esposito and Silva 1998). Se consideran hongos cosmopolitas del suelo, son generalmente aeróbicos y, en determinadas condiciones, pueden ser anaerobios facultativos, lo que les permite mostrar una mayor plasticidad ecológica (Rahman et al. 2011). Las necesidades nutricionales de Trichoderma son bien conocidas: son capaces de degradar sustratos 
muy complejos como celulosa, quitina, almidón, xilano y pectina. Gracias al gran complejo enzimático que poseen, las especies de Trichoderma pueden utilizar dichos sustratos para su crecimiento. Asimismo, asimilan como fuente de nitrógeno compuestos tales como aminoácidos, urea, nitritos, amoniaco y sulfato de amonio (Agamez Ramos et al. 2008). Todas estas características nutricionales, fisiológicas y adaptativas hacen que las especies de Trichoderma sean muy eficientes como agentes de control biológico (Stocco et al. 2016). El género Trichoderma fue descripto por primera vez por Persoon en 1794, pero capturó la atención de los agricultores sólo después de que Weindling et al. (1934) mostraron que una especie del género pudo eliminar a otros hongos, y controlar las enfermedades de las plantas. Los mecanismos antagónicos que utilizan las especies de Trichoderma se describen como competencia por nutrientes, por oxigeno o por espacio, antibiosis mediante la producción de metabolitos volátiles y no volátiles, y micoparasitismo directo con producción de enzimas extracelulares o metabolitos secundarios, sin ser estos mecanismos mutuamente excluyentes (Infante 2009). Además, las especies de Trichoderma generan promoción de crecimiento e inducción de resistencia en la planta huésped (Infante 2009). Dicha comunidad microbiana es conocida y explorada a nivel mundial por sus diversas aplicaciones en la agricultura. En el mercado internacional la cantidad de productos que contienen Trichoderma ha crecido exponencialmente en los últimos cinco años, con más de 250 productos disponibles (Woo et al. 2014). En Brasil, país líder mundial en el sector de los agronegocios, según el Ministerio de la Agricultura, Pecuária y Abastecimento (MAPA), se registraron 133 biopesticidas, de los cuales el $43 \%$ es a base de Trichoderma spp. En este país el área tratada con Trichoderma en los últimos años aumentó a más de 3 millones de hectáreas (Bettiol et al. 2012). En Chile actualmente existen 17 productos biológicos a base de Trichoderma, los cuales se comercializan y se encuentran oficialmente registrados en el Servicio Agrícola y Ganadero; Trichonativa es el producto que se recomienda para manejar enfermedades (Lolas and Sandoval 2013). En 2012, en Colombia se reportaron 12 productos con capacidad biocontroladora de plagas registrados ante la Agencia de Protección Ambiental (EPA). En Estados Unidos de América (EUA) se registraron bioinsumos a base de Trichoderma asperellum (Hypocreales:
Hypocreaceae) (Samuels et al. 1999), T. harzianum (Hypocreales: Hypocreaceae) (Rifai 1969) y Trichoderma polysporum (Hypocreales: Hypocreaceae) (Rifai 1969) (Howel et al. 2003). Perú cuenta con un programa Nacional de Control Biológico el cual logró incrementar el empleo del biocontroladores de 10000 ha/año a 253000 ha/año en un período de seis años, con un ahorro del 50\% comparado con el uso de pesticidas en 17 cultivos de importancia económica en el país (Cueva 2012). Además, en Perú se han desarrollaron más de 50 empresas productoras de controladores biológicos a base de Trichoderma, entre otros microorganismos (Cueva 2012). Se han registrado productos a base de Trichoderma en países de la Unión Europea (Bélgica, Chipre, Francia, Italia, Países Bajos, España, Suecia, Eslovenia y el Reino Unido), en América del Norte (EUA y Canadá), África (Sudáfrica y Marruecos), Asia (India y Vietnam) y en Oceanía (Australia y Nueva Zelanda) (Woo et al. 2014). La mayor distribución de bioproductos de Trichoderma se encuentra en Asia, seguida por Europa, América Central y América del Sur (Woo et al. 2014). En el mercado argentino existen actualmente alrededor de 14 bioproductos cuyos principios activos son cepas de Trichoderma, siendo las especies más utilizadas para el control biológico $T$. harzianum, $T$. koningii (Hypocreales: Hypocreaceae) (Rifai 1969), Trichoderma atroviride (Hypocreales: Hypocreaceae) (Bissett 1984) y Trichoderma koningiopsis (Hypocreales: Hypocreaceae) (Samuels et al. 2006) (Tabla 1).

\section{Estudios sobre control biológico aplicando Trichoderma en la agricultura argentina}

En la Argentina, la actividad agrícola es uno de los pilares fundamentales de la economía, ya que su extenso territorio y la variedad climática existente permite el desarrollo de distintos cultivos (Reca and Parellada 2001). Actualmente, el país se identifica con la producción de granos, como son el trigo, el maíz, el girasol y la soja. Esto se debe a la superficie dedicada, las condiciones de la tierra y a los ingresos que generan sus exportaciones. Los productos hortícolas, por su parte, tienen la posibilidad de permanecer constantes en el mercado durante todo el año, lo que determina su importancia social y económica a través de una contribución decisiva para la alimentación dela población(Fernández 2012). Por otra parte según un informe del Ministerio de Ciencia, Tecnología e Innovación Productiva sobre el perfil sectorial de la producción de frutas 
Tabla 1. Productos comerciales a base de formulaciones sólidas de Trichoderma en la Argentina. Table 1. Commercial products based on solid formulations of Trichoderma in Argentina.

\begin{tabular}{|c|c|c|c|c|}
\hline Nombre comercial & Principio activo & Función & Lugar & Empresa \\
\hline Cero Pestes Cultivo & T. viride & Fungicida & C.A.B.A & Sanipro \\
\hline Trichoderma sp. & T. viride & Fungicida & Tucumán & Laboratorios San Pablo \\
\hline Trichoderma & Trichoderma sp. & Larvicida-Fungicida & $\begin{array}{l}\text { Ramos Mejía } \\
\text { (Bs. As.) }\end{array}$ & Ecomambo \\
\hline Rizoderma* & T. harzianum & Terapéutico de semillas & $\begin{array}{l}\text { Pergamino } \\
\text { (Bs. As.) }\end{array}$ & Rizobacter \\
\hline Trichoderma Tifi & T. atroviride & Fungicida & $\begin{array}{l}\text { Recoleta } \\
\text { (Bs. As.) }\end{array}$ & How High Grow Shop \\
\hline Trichoderma & T. harzianum & Fungicida & $\begin{array}{l}\text { Olivos } \\
\text { (Bs. As.) }\end{array}$ & Olivos Grow Shop \\
\hline Trichomix spp. & T. harzianum & Fungicida & $\begin{array}{l}\text { San Fernando } \\
\text { (Bs. As.) }\end{array}$ & $\begin{array}{l}\text { Organix Argentina } \\
\text { Biotecnología }\end{array}$ \\
\hline Minitric & T. harzianum & $\begin{array}{l}\text { Biocontrolador, biofertilizante, } \\
\text { bioestimulante }\end{array}$ & C.A.B.A & La Juana Grow Shop \\
\hline Tricho Grow & Trichoderma sp. & $\begin{array}{l}\text { Fungicida, promotor de } \\
\text { crecimiento }\end{array}$ & $\begin{array}{l}\text { Recoleta } \\
\text { (Bs. As.) }\end{array}$ & Biogood \\
\hline Power Zyme de Hesi & T. viride & Fungicida & $\begin{array}{l}\text { Banfield } \\
\text { (Bs.As.) }\end{array}$ & Amira Grow \\
\hline Tch Trichoderma & T. harzianum & Biocontrolador, fungicida & C.A.B.A. & $\begin{array}{l}\text { Arcadia Grama } \\
\text { Growshop }\end{array}$ \\
\hline Tricofull & Trichoderma sp. & Fertilizante, bioestimulante & C.A.B.A & Brometan \\
\hline Tratbac & T. harzianum & Fertilizante, bioestimulante & $\begin{array}{l}\text { Ramos Mejía } \\
\text { (Bs. As.) }\end{array}$ & Bea Tecno Bio S.A. \\
\hline Tricho Seeds & Trichoderma sp. & $\begin{array}{l}\text { Potenciador de germinación, } \\
\text { fungicida }\end{array}$ & C.A.B.A. & Compañía Orgánica \\
\hline
\end{tabular}

*Producto comercial inscripto y aprobado por el SENASA.

cítricas, la Argentina es el octavo productor mundial de cítricos, el primer productor mundial de limón y el segundo por cantidad de limones frescos exportados (Schliserman and Ovruski 2004). Es interesante también el cultivo de vid en nuestro país, que se utiliza para varios fines. Sus frutos fermentados se transforman en vinos y brandy. Por otra parte se consume dicho fruto tanto en fresco como en pasas (seco) durante todo el año. Otros usos comunes del fruto en diferentes mercados son, jugo no fermentado, jugo concentrado helado y conservas (Pearson and Goheen 1996). En lo que respecta a los cultivos de interés industrial, la Argentina es el primer productor mundial y consumidor de yerba mate, responsable del $62 \%$ de la producción en el mundo (Traglia et al. 2018).

Existe una amplia variedad de hongos patógenos que afectan el normal desarrollo y la producción de todos estos cultivos. Los daños en el rendimiento de la producción oscilan entre el $17 \%$ y el $50 \%$ dependiendo del estado fenológico en el que ocurre la infección. En algunos casos extremos, las pérdidas podrían alcanzar el $95 \%$ de la producción en determinados campos (Oddino et al. 2018). Debido a esto, los tratamientos con fungicidas no son lo suficientemente efectivos para controlar las enfermedades en estos cultivos, por lo que la búsqueda y el empleo de estrategias eficaces para el control de estos microorganismos podría contribuir en una agricultura sostenible, económica y ambientalmente saludable. Los primeros estudios sobre control biológico de hongos fitopatógenos en la Argentina se iniciaron a mediados del siglo XX. El primer curso de posgrado Control Biológico de Enfermedades de las Plantas fue dictado en Buenos Aires en 1996 por el Dr. John Sutton (University of Guelph, Canadá), quien transmitió su entusiasmo a numerosos investigadores. En 1998 se realizó en Buenos Aires el $1^{\circ}$ Congreso Argentino de Control Biológico de Enfermedades de las Plantas; lamentablemente, no tuvo continuidad. Hasta el momento no publicaron libros sobre esta temática, sólo recopilaciones de trabajos realizados (Rivera and Wright 2014). A lo largo de los años fueron en incremento las publicaciones en revistas, jornadas y congresos de los trabajos de investigación con objetivos 
Tabla 2. Estudios llevados a cabo y publicados sobre control biológico de fitopatógenos con cepas de Trichoderma en la Argentina.

Table 2. Studies carried out and published on biological control of phytopathogen with strains of Trichoderma in Argentina.

\begin{tabular}{|c|c|c|c|c|}
\hline Especies & Cultivo & Acción & Referencia & Lugar \\
\hline T. koningii; T. viride & Hortalizas & Inhibición de S. sclerotiorum y Sarracenia minor & Wright et al. (1988) & Buenos Aires \\
\hline T. koningii & G. $\max$ (soja) & Antagonismo frete a Colletotrichum dematium & Grijalba et al. (1992) & Buenos Aires \\
\hline $\begin{array}{l}\text { Trichoderma } \\
\text { pseudokoningii; } \\
\text { Trichoderma hamatum }\end{array}$ & Hortalizas & $\begin{array}{l}\text { Antagonismo frente a Fusarium solani, } \\
\text { Fusarium equiseti, F. oxysporum, Rhizoctonia sp., } \\
\text { S. sclerotiorum, S. minor y S. rolfsii }\end{array}$ & Mónaco et al. (1994) & La Plata \\
\hline T. koningii & T. aestivum (trigo) & Inhibición de B. sorokiniana & Dal Bello et al. (1994) & La Plata \\
\hline T. harzianum & Hortalizas & Producción de un formulado líquido & Cozzi y Gasoni (1995) & Buenos Aires \\
\hline Trichoderma sp. & $\begin{array}{l}\text { L. sativa (lechuga); } \\
\text { Cichorium endivia } \\
\text { (endivia) }\end{array}$ & $\begin{array}{l}\text { Producción de formulados líquidos y sólidos } \\
\text { aplicadas contra } S \text {. sclerotiorum y } R \text {. solani }\end{array}$ & Zapata et al. (1997) & Buenos Aires \\
\hline T. harzianum & G. $\max (\mathrm{soja})$ & $\begin{array}{l}\text { Inhibición de S. sclerotiorum. Determinación } \\
\text { enzimática }\end{array}$ & $\begin{array}{l}\text { Menéndez y Godeas } \\
\text { (1998) }\end{array}$ & La Plata \\
\hline Trichoderma sp. & S. lycopersicum (tomate) & Antagonismo frente a $R$. solani & Durman et al. (1999) & Buenos Aires \\
\hline Trichoderma spp. & $\begin{array}{l}\text { Hordeum vulgare } \\
\text { (cebada) }\end{array}$ & Antagonismo frente a S. sclerotiorum & Cúndom et al. (2002) & Corrientes \\
\hline T. harzianum & T. aestivum (trigo) & Inhibición de D. tritici-repentis & Perello et al. (2003) & La Plata \\
\hline Trichoderma spp. & Hortalizas & Inhibición de $R$. solani & $\begin{array}{l}\text { Mazza de Gaiad et al. } \\
\text { (2003) }\end{array}$ & Corrientes \\
\hline T. harzianum & Arachis hypogaea (maní) & Inhibición de F. solani & Rojo et al. (2006) & Córdoba \\
\hline $\begin{array}{l}\text { T. harzianum; } T \text {. } \\
\text { longibrachiatum }\end{array}$ & Zea mays (maíz) & Inhibición de F. proliferatum & Rojo et al. (2007) & Córdoba \\
\hline T. harzianum & Beta vulgaris (remolacha) & )Inhibición de R. solani & Gasoni et al. (2008) & Córdoba \\
\hline Trichoderma sp. & Vitis vinifera (vid) & Inhibición de $B$. cinérea & Lucero et al. (2008) & Mendoza \\
\hline T. harzianum & T. aestivum (trigo) & Inhibición de Septoria tritici & Perello et al. (2009) & La Plata \\
\hline Trichoderma spp. & $V$. vinífera (vid) & Inhibición de $B$. cinerea & Hapon et al. (2010) & Mendoza \\
\hline $\begin{array}{l}\text { T. koningii; T. virens; } T \text {. } \\
\text { harzianum }\end{array}$ & S. lycopersicum (tomate) & Antagonismo frente F. oxysporum & Colombo et al. (2011) & Corrientes \\
\hline T. harzianum & T. aestivum (trigo) & Inhibición de B. sorokiniana y Pyricularia oryzae & Consolo et al. (2012) & Mar del Plata \\
\hline Trichoderma sp. & S. lycopersicum (tomate) & $\begin{array}{l}\text { Inhibición de F. oxysporum, R. solani, F. } \\
\text { subglutinans y Rhizoctonia sp. }\end{array}$ & $\begin{array}{l}\text { Barocio Ceja et al. } \\
\text { (2013) }\end{array}$ & Buenos Aires \\
\hline Trichoderma spp. & Hortalizas & $\begin{array}{l}\text { Antagonismo frente Alternaria sp., Pythium } \\
\text { sp., Phytophtora sp., Botrytis sp., F. oxysporum y } \\
\text { Pestalotia sp.; tolerancia a fungicidas químicos }\end{array}$ & $\begin{array}{l}\text { Gutiérrez Brower et } \\
\text { al. (2013) }\end{array}$ & Misiones \\
\hline Trichoderma sp. & S. lycopersicum (tomate) & Inhibición de F. oxysporum & Flores et al. (2014) & Jujuy \\
\hline Trichoderma sp. & $V$. vinífera (vid) & Inhibición de Phytophthora capsici & Lucero et al. (2014) & Mendoza \\
\hline Trichoderma spp. & $\begin{array}{l}\text { Brassica juncea, (mostaza } \\
\text { castaña) }\end{array}$ & Inhibición de F. graminearum & Perniola et al. (2014) & Buenos Aires \\
\hline T. harzianum & G. $\max ($ soja) & Inhibición Fusarium spp. y Phomopsis spp. & $\begin{array}{l}\text { Crovo y Clemente } \\
(2015)\end{array}$ & Santa Fe \\
\hline $\begin{array}{l}\text { T. koningiopsis, } T \text {. } \\
\text { atroviride }\end{array}$ & A. cepa (cebolla) & Inhibición de F. oxysporum & Sánchez et al. (2015) & La Plata \\
\hline T. harzianum & S. lycopersicum (tomate) & Inhibición de Clavibacter michiganensis & Guerrero (2016) & La Plata \\
\hline T. harzianum & T. aestivum (trigo) & Inhibición de M. graminicola & Stocco et al. (2016) & La Plata \\
\hline T. viride & G. $\max$ (soja) & Inhibición de Macrophonia phaseolina & Reznikov et al. (2016) & Tucumán \\
\hline Trichoderma sp. & Fragaria (frutilla) & Inhibición de $B$. cinerea & Murillo et al. (2016) & Salta \\
\hline T. harzianum & A. hypogaea (maní) & $\begin{array}{l}\text { Preparación de un formulado liquido contra } \\
\text { Thecaphora frezii }\end{array}$ & Ganuza et al. (2016) & Córdoba \\
\hline Trichoderma sp. & S. lycopersicum (tomate) & Inhibición de $R$. solani & $\begin{array}{l}\text { Sánchez y Gassó } \\
\text { (2017) }\end{array}$ & Buenos Aires \\
\hline $\begin{array}{l}\text { T. konigiopsis; } T \text {. } \\
\text { atroviride }\end{array}$ & $\begin{array}{l}\text { A. sativum (ajo); A. cepa } \\
\text { (cebolla) }\end{array}$ & Antagonismo frente S. cepivorum & Conles et al. (2017) & Córdoba \\
\hline Trichoderma sp. & G. $\max$ (soja) & Inhibición de M. phaseolina & \multicolumn{2}{|c|}{ Maumary et al. (2017)Santa Fe } \\
\hline Trichoderma sp. & M. domestica (manzana) & Antagonismo frente a Valsa ceratosperma & Valetti et al. (2017) & Río Negro \\
\hline Trichoderma sp. & P. persica (durazno) & Antagonismo frente a $M$. fructicola & \multicolumn{2}{|c|}{ Alvarado et al. (2017) Mendoza } \\
\hline Trichoderma sp. & $\begin{array}{l}\text { I. paraguariensis (yerba } \\
\text { Mate) }\end{array}$ & $\begin{array}{l}\text { Antagonismo frente a Alternaria destruens, } \\
\text { Phoma sp., Colletotrichum gigasporum y F. } \\
\text { oxysporum }\end{array}$ & $\begin{array}{l}\text { Vereschuk et al. } \\
\text { (2017) }\end{array}$ & Misiones \\
\hline $\begin{array}{l}\text { T. harzianum; T. } \\
\text { koningiopsis; T. atroviride }\end{array}$ & $e^{\text {A. hypogaea (maní) }}$ & Antagonismo frente a S. minor y S. rolfsii & $\begin{array}{l}\text { Rodríguez et al. } \\
\text { (2017) }\end{array}$ & Córdoba \\
\hline Trichoderma sp. & $\begin{array}{l}\text { Nicotiana tabacum } \\
\text { (tabaco) }\end{array}$ & Inhibición de $R$. solani & Harries et al. (2017) & Salta \\
\hline Trichoderma sp. & G. $\max ($ soja) & Inhibición de M. phaseolina & Vogrig et al. (2017) & Corrientes \\
\hline
\end{tabular}


específicos sobre cepas de Trichoderma contra diversos patógenos, extendiéndose dichos estudios sobre diferentes cultivos a lo largo de todo el territorio argentino.

Las especies de Trichoderma poseen diferentes roles en la agricultura argentina, por lo que cerca de 50 grupos, que representan a varias universidades e institutos de investigación de nuestro país, están trabajando en distintas aplicaciones de Trichoderma como controlador biológico. Hasta la actualidad se describieron cerca de 10 especies diferentes de Trichoderma con capacidad antagonista y se han estudiado alrededor de 35 cultivos y 40 patógenos (Tabla 2).

A partir del análisis de la información recopilada inferimos que la especie de Trichoderma más estudiada como agente de control biológico en la Argentina es $T$. harzianum seguida de $T$. koningii y $T$. viride (Hypocreales: Hypocreaceae) (Persoon 1794). Estas cepas tienen la capacidad de actuar sobre un amplio espectro de fitopatógenos a través de sus diversos mecanismos de acción. A T. harzianum se le atribuye un gran éxito de comercialización como producto biológico en muchos lugares del mundo, aproximadamente el 50\% de los bioproductos registrados son a base de esta especie. Por su parte, T. viride es una especie que se utiliza en gran medida como agente de control biológico en Asia, particularmente en India, donde se encuentra en casi el $70 \%$ de los formulados disponibles (Sharma et al. 2014).

Uno de los cultivos afectados por hongos fitopatógenos sobre los que se desarrolla la mayoría de las investigaciones en la Argentina es la soja (Glycine max, Fabales: Fabaceae) (Merrill 1917), cultivo que ocupa el 60\% del área sembrada del país. El poroto de soja y sus derivados (e.g., pellets, aceites) constituyen el principal rubro de exportación de la Argentina, uno de los países productores líderes a nivel mundial (Aizen 2009). Este cultivo es afectado principalmente por Sclerotinia sclerotiorum (Helotiales: Sclerotiniaceae) (Bary 1884.), Macrophomina phaseolina (Botryosphaeriales: Botryosphaeriaceae) (Goidánich 1947) y Rhizoctonia spp. (Agonomicetales: Agonomicetaceae) (De Candolle 1815). Según las investigaciones llevadas a cabo hasta el momento, cepas de T. harzianum se han reportado como buenas controladoras de dichos fitopatógenos. El tomate (Solanum lycopersicum, Solanales: Solanaceae) (Linneo 1753), representa uno de los cultivos hortícolas más importantes a lo largo de la Argentina (Carluccio et al. 2002). Este cultivo está afectado principalmente por Fusarium oxysporum (Hypocreales: Nectriaceae) (Wollenweber 1930), Rhizoctonia solani (Agonomicetales: Agonomicetaceae) (Schultz 1936) y S. sclerotiorum. Las cepas de T. harzianum, $T$. viride y $T$. koningii se han reportado como buenas controladoras de dichos fitopatógenos. El trigo (Triticum aestivum, Poales: Poaceae) (Linneo 1753), el cual ha sido a lo largo de su historia uno de los principales actores en el comercio internacional de la Argentina (Miralles and González 2010), se ve afectado principalmente por Bipolaris sorokiniana (Pleosporales: Pleosporaceae) (Shoemaker 1959) y Drechslera tritici-repentis (Pleosporales: Pleosporaceae) (Shoemaker 1962). Cepas de T. harzianum se han reportado como buenas controladoras de dichos fitopatógenos. La lechuga (Lactuca sativa, Asterales: Asteraceae) (Linneo 1753), la cual ocupa el tercer lugar dentro de las hortalizas cultivadas en la Argentina, encontrándose en casi todo el país en sus distintas variedades (Katinas et al. 2007). Este cultivo es afectado principalmente por $S$. sclerotiorum y R. solani. Cepas de T. koningii y Trichoderma afroharzianum (Hypocreales: Hypocreaceae) (Chaverri et al. 2015) se han reportado como buenas controladoras de dichos fitopatógenos. El ajo (Allium sativum, Asparagales: Amaryllidaceae) (Linneo 1753), cultivo que ubica al país como el segundo exportador a nivel mundial y uno de los principales productores (Burba and Galmarini 1994). Esta afectado principalmente por Sclerotium cepivorum (Helotiales: Sclerotiniaceae) (Berk 1842) y Fusarium sp. Cepas de T. koningiopsis y T. harzianum se han reportado como buenas controladoras de dichos fitopatógenos. En cuanto a la cebolla (Allium cepa, Asparagales: Amaryllidaceae) (Linneo 1753), con una producción anual de 450 millones de toneladas, la Argentina es el principal productor y exportador de cebolla deshidratada de América Latina y posee un promedio de consumo per cápita de la hortaliza fresca de $10 \mathrm{~kg}$ al año (Jaldo and Delia 2017). Esta demanda del mercado interno se encuentra totalmente abastecida por la producción nacional. Cepas de $T$. koningiopsis y $T$. atroviride se han reportado como buenas controladoras de F. oxysporum en el cultivo de cebolla.

En relación con los hongos fitopatógenos más investigados en la Argentina, según nuestra recopilación de información se encuentran 
Fusarium sp., un extenso género de hongos filamentosos, ampliamente distribuido en el suelo y en asociación con muchas enfermedades de las plantas. Son productores de micotoxinas, capaces de desencadenar alteraciones y cuadros patológicos en diversos cultivos. Las principales enfermedades causadas por este hongo reportadas en nuestro país son: el tizón de cereales; la pudrición de las raíces en cosecha de papa, tomate, yerba mate y la marchitez en plantas ornamentales (Abarca et al. 2000). Los distintos grupos de trabajo aplican diversas cepas de Trichoderma sp., siendo la más favorable para el control biológico de este hongo T. harzianum. Otro patógeno fúngico ampliamente estudiado en la Argentina es el género Sclerotium sp., habitante del suelo, con un amplio conjunto de huéspedes. La enfermedad producida por este hongo recibe muchas denominaciones diferentes en la literatura: pudrición basal de tallo y raíz, mal del esclerocio, añublo sureño o tizón sureño, son algunas de ellas (Singh and Mathur 1974). Las especies de Trichoderma que se aplican con mayor frecuencia para el control de Sclerotium sp. son T. harzianum y $T$. atroviride predominantemente en cultivos de lechuga, cebolla, tomate, ajo y girasol. Otros fitopatógenos estudiados hasta el momento en menor frecuencia pero no menos importantes son Botrytis sp. (Helotiales: Sclerotinicaeae) (Haller 1768), Rhizoctonia sp., Pythium sp. (Pythiales: Pythiaceae) (Pringsheim 1858), Phytophtora sp. (Peronosporales: Peronosporaceae) (Bary 1876), Drechslera sp., Verticillum sp. (Hypocreales: Plectosphaerellaceae) (Nees 1817.), Phoma sp. (Pleosporales: Didymellaceae) (Saccardo 1880), Macrophomina sp. y Alternaria sp. (Pleosporales: Pleosporaceae) (Nees 1817), entre otros.

Podemos destacar que el modo de acción más estudiado de Trichoderma en la agricultura argentina es la inhibición que ejerce sobre diversos patógenos fúngicos. Esta se lleva a cabo por antibiosis que ocurre durante interacciones que involucran la producción de antibióticos que inhiben el crecimiento de otros microorganismos (Benítez et al. 2004). Los ensayos de antagonismo también han sido muy numerosos en el transcurso de los años en nuestro país. Dicho mecanismo de acción se da por el micoparasitismo, en el que se produce un ataque directo de un hongo sobre otro, proceso muy complejo que involucra eventos secuenciales que incluyen reconocimiento, ataque y penetración de las hifas del antagonista y posterior muerte del microorganismo huésped (Benítez et al. 2004). Se han desarrollado también diversos estudios sobre la generación de bioformulados a base de Trichoderma. Aproximadamente el $90 \%$ de los actuales micoplaguicidas para control biológico de hongos fitopatógenos tiene como principio activo las esporas (conidios y clamidiosporas) de Trichoderma spp. (Pineda Insuasti et al. 2017). La mayor parte de los productos comercializados están compuestos, según la etiqueta, de una única especie de Trichoderma (67\%). Sin embargo, la generación de productos biológicos con diferentes combinaciones de Trichoderma han demostrado mejores resultados (Sharma et al. 2014). Las mayores limitaciones del uso y comercialización de una formulación adecuada son asegurar su fácil aplicación, bajar los costos de producción, obtener una concentración suficiente de inóculo, una alta viabilidad de las esporas y mantener la densidad de inóculo una vez aplicado en el suelo o sustrato, tras un período de tiempo de medio a largo plazo (Viera et al. 2018).

\section{Conclusiones}

Actualmente, el género Trichoderma es uno de los agentes de mayor uso en programas de MIP como antagónico y regulador de hongos fitopatógenos. Se han realizado numerosos estudios de control biológico de enfermedades de las plantas en la Argentina, tanto básicos como aplicados. Dado lo extenso del país, donde se presentan diferentes condiciones edafo-climáticas, se necesitan aplicar estrategias de manejo particulares, estudiando principalmente cepas nativas, ya adaptadas a sus condiciones ambientales. Si bien se puede observar cierta concentración de estudios en algunas zonas, en los últimos años se destaca la realización de investigaciones en gran parte del territorio y sobre producciones diversas, que abarcan desde la iniciación del cultivo hasta la postcosecha. Se han realizado avances muy importantes, aunque en casi todos los casos no se ha llegado a una etapa de registro y comercialización.

En la actualidad, en la Argentina hay 3958 formulados inscriptos en el Servicio Nacional de Sanidad y Calidad Agroalimentaria, entre fungicidas, antibióticos, insecticidas, herbicidas, acaricida y nematicidas. Se contabilizan 965 registros de actividad fungicida y 15 de actividad bactericida, de los cuales sólo 160 son de origen orgánico. Entre estos hay registrado un solo formulado a base de T. harzianum. No hay ningún producto 
biológico registrado para la línea jardín, incluyendo formulados aplicables a nivel de pequeñas superficies implantadas con especies ornamentales. La meta de la investigación en esta área es contribuir al desarrollo agrícola en forma sostenible y en armonía con la naturaleza utilizando herramientas como el control biológico. Éste podría sustituir o disminuir el uso de agroquímicos que se aplican actualmente en concentraciones que son perjudiciales a la salud humana y dañan al medio ambiente.

Mediante el fortalecimiento de instituciones y el apoyo a la investigación se puede lograr una manipulación exitosa de antagonistas a través del control biológico dando como resultado una práctica agrícola económicamente viable y de bajo impacto ambiental. A partir de futuros estudios in vivo de las cepas más eficientes, la profundización en estudios de ecología microbiana y la especialización en aspectos asociados a la tecnología de formulación, se podrá avanzar en el desarrollo de bioinsumos comerciales. Éstos, además de crear una importante vía para el desarrollo de una nueva industria nacional, de carácter biotecnológico, podrían proporcionar soluciones eficaces a los principales problemas patológicos que afectan al sector agrícola en el país.

\section{REFERENCIAS}

Abarca, M. R., M. R. Bragulat, G. Castellá, F. Accensi, and F. J. Cabañes. 2000. Hongos productores de micotoxinas emergentes. Revista Iberoamericana de Micología 17:S63-S68.

Agamez Ramos, E., R. Zapata Navarro, L. Oviedo Zumaque, and J. Barrera Voleth. 2008. Evaluación de sustratos y procesos de fermentación sólida para la producción de esporas de Trichoderma sp. Revista Colombiana de Biotecnología 10:23-34.

Aizen, M. A., L. A. Garibaldi, and M. Dondo. 2009. Expansión de la soja y diversidad de la agricultura argentina. Ecología Austral 19:45-54.

Altieri, M., and C. I. Nicholls. 2000. Agroecología. Teoría y práctica para una agricultura sustentable. Serie Textos Básicos para la Formación Ambiental. $1^{\circ}$ Edición. Programa de las Naciones Unidas para el Medio Ambiente Red de Formación Ambiental para América Latina y el Caribe, Colonia Lomas de Virreyes, México D.F., México. Pp. 167-180.

Alvarado, P., J. J. Boiteux, C. Monardez, R. López Plantey, G. Lucero, and P. Pizzuolo. 2017. Actividad antimicrobiana de filtrados culturales de distintos aislados de Trichoderma sp. hacia Monilinia fructicola. Resumen $4^{\circ}$ Congreso Argentino de Fitopatología. Asociación Argentina de Fitopatólogos, Mendoza, Argentina. Pp. 361. URL: https: /tinyurl.com/scqx3xp

Barocio Ceja, N. B., L. F. Ceja Torres, J. L. Morales García, H. V. Silva Rojas, R. Flores Magallón, and S. Y. Ochoa Estrada. 2013. In vitro biocontrol of tomato pathogens using antagonists isolated from chicken-manure vermicompost. Phyton 82:15-22.

Bary, A. 1876. Researches into the nature of the potato-fungus, Phytophthora infestans. Journal of the Royal Agricultural Society of England 12:239-269.

Bary, A. 1884. Vergleichende Morphologie und Biologie der Pilze Mycetozoen und Bacterien. Wilhelm Engelmann Pp. 1-558. https://doi.org/10.5962/bhl.title.42380.

Benítez, T., A. M. Rincón, M. C. Limón, and A. C. Codón. 2004. Biocontrol mechanisms of Trichoderma strains. International Microbiology 7:249-260.

Berk, A. 1842. The Annals and Magazine of Natural History 6:359.

Bettiol, W. 2006. Productos alternativos para el manejo de enfermedades en cultivos comerciales. Fitosanidad 10: 85-98.

Bettiol, W., M. A. Morandi, Z. V. Pinto, T. J. Paula Junior, E. B. Correa, A. B. Moura, C. M. Lucon, J. C. Costa, and J. L. 2012. Produtos comerciais à base de agentes de biocontrole de doenças de plantas. Embrapa Meio Ambiente, Jaguariuna, Jaguariúna, São Paulo, Brasil.

Bettiol, W., and R. Ghini. 2014. Proteção de plantas em sistemas agrícolas alternativos. Cadernos de Ciência y Tecnologia 17:61-70.

Burba, J. L., and C. R. Galmarini. 1994. Allium crop situation in Argentina. En I International Symposium on Edible Alliaceae 433:35-52. https: / / doi.org/10.17660/ActaHortic.1997.433.2.

Carluccio, C., M. Lenscak, M. Panelo, M. Colombo, S. Cáceres, N. Molina, and C. Pernuzzi. 2002. Desarrollo actual de los cultivos protegidos en la República Argentina. Situación de la Agroplasticultura en Países Iberoamericanos. Tercera Reunión de Coordinación en Caracas, Venezuela. Cyted, Almería, España. Septiembre. Pp. 30-71.

Chaverri, P., F. B. Rocha, W. Jaklitsch, R. Gazis, T. Degenkolb, and G. J. Samuels. 2015. Systematics of the Trichoderma harzianum species complex and the re-identification of commercial biocontrol strains. Mycologia 107:558-590. https: //doi.org/10.3852/14-147.

Colombo, M. H., T. Lattar, V. Obregón, N. Cardozo, J. Monteros, and C. Mónaco. 2011. Acción antagónica in vitro de T. koningii, T. virens y T. harzianum sobre Fusarium oxysporum $\mathrm{f}$ sp. lycopersici patógeno del tomate en invernaderos plásticos. Horticultura Argentina 30:53-73.

Conles, M., A. Pérez, M. Blengini, I. Bochetto, M. Balzarini, C. Pinotti, and J. Muñoz. 2017. Antagonismo de aislamientos nativos de Trichoderma sobre Sclerotium cepivorum. Resumen $4^{\circ}$ Congreso Argentino de Fjtopatología. Asociación Argentina de Fitopatólogos, Mendoza, Argentina. Pp. 3113. URL: https://tinyurl.com/scqx3xp.

Consolo, V., C. Mónaco, C. Cordo, and G. Salerno. 2012. Characterization of novel Trichoderma spp. isolates as a search for effective biocontrollers of fungal diseases of economically important crops in Argentina. World Journal of Microbiology and Biotechnology 28:1389-1398. https:// doi.org/10.1007/s11274-011-0938-5.

Cook, R. J., and K. F. Baker. 1983. The Nature and Practice of Biological Control of Plant Pathogens. American Phytopathological Society. Pp. 539. 
Cozzi, J., and L. Gasoni. 1995. Producción de biomasa de Trichoderma harzianum en distintos medios y condiciones de cultivo. Revista forestal Venezolana 1:28.

Crovo, V., and G. Clemente. 2015. Eficacia de fungicidas de síntesis y biológico en base a Trichoderma harzianum para controlar patógenos de semillas de soja. FAVE. Sección Ciencias agrarias 14:666-7719. https://doi.org/10.14409/ fa.v14i2.5720.

Cueva, F. D. 2012. El control biológico como estrategia para apoyar las exportaciones agrícolas no tradicionales en Perú: un análisis empírico. Contabilidad y Negocios 7:81-100.

Cúndom, M. A., S. M. Maza, M. A. Mazzanti, S. A. Gutiérrez, and M. Couthino. 2002. Actividad antagónica in vitro de hongos saprófitos del departamento Capital, provincia de Corrientes, Argentina, contra Sclerotinia sclerotiorum. Fitopatología 37:133-141.

Dal Bello, G., C. Mónaco, and M. Sisterna. 1994. Efecto de Trichoderma spp. sobre el control del tizón de la plántula en trigo ocasionado por Bipolaris sorokiniana bajo condiciones de invernáculo. Fitopatologia Brasileira 19:394-400.

DeBach, P. 1964. Successes, trends, and future possibilities. Pp. 673-713 in P. DeBach (ed.). Biological Control of Insect Pests and Weeds. Reinhold, N.Y. Pp. 844.

De Candolle, A. P. 1815. Flore Française 6:1-662.

Durman, S., A. Méndez, and A. Godeas. 1999. Evaluación de Trichoderma spp. como antagonista de Rhizoctonia solani in vitro y como biocontrolador del damping-off de plantas de tomates en invernadero. Revista Argentina de Microbiología 31:13-18.

Esposito, E., and M. D. Silva. 1998. Systematics and environmental application of the genus Trichoderma. Critical Reviews in Microbiology 24:89-98. https://doi.org/10.1080/10408419891294190.

Etchegoyen, M. A., A. E. Ronco, and P. Almada. 2017. Occurrence and fate of pesticides in the Argentine stretch of the Paraguay-Paraná basin. Environmental Monitoring and Assessment 189:63. https://doi.org/10.1007/s10661017-5773-1.

Fravel, D. 2005. Commercialization and implementation of biocontrol. Annual Review of Phytopathology 43:1-23. https: / / doi.org/10.1146/annurev.phyto.43.032904.092924.

Fernández, J. 2012. La producción de hortalizas en Argentina. Corporación del Mercado Central de Buenos Aires. Pp. 29.

Flores, C. R., S. G. Bejarano, N. M. Rueda, R. E. Rueda, and D. Flores Alzaga. 2014. Cepas de Trichoderma B-69; B-67 y B-72 seleccionadas por su actividad supresiva sobre la podredumbre vascular del tomate. Resumen XXXVII Congreso Argentino de Horticultura 33:57.

Ganuza, M. R., N. A. Pastor, J. Folguera, J. Andrés, M. M. Reynoso, M. Rovera, and A. M. Torres. 2016. Perspectiva de aplicación de bioformulado a base de Trichoderma harzianum ítem 3636 para el control de carbón de maní. Plant Disease 101:400-408.

Gasoni, L., N. Kahn, V. Yossen, J. Cozzi, K. Kobayashi, S. Babbitt, V. Barrera, and G. Zumelzú. 2008. Effect of soil solarization and biocontrol agents on plant stand and yield on table beet in Córdoba (Argentina). Crop Protection 27:337-342. https://doi.org/10.1016/j.cropro.2007.06.004.

Goidánich, G. 1947. Revisione del genere Macrophomina Petrak. Specie tipica: Macrophomina phaseolina (Tassi) G. Goid. n. comb. nec M. phaseoli (Maubl.) Ashby. Annali della Sperimentazione Agaria 1:449-461.

Guerrero, R. 2016. Selección y efectividad del uso de aislamientos de Trichoderma spp. para el control del Cancro Bacteriano del tomate (Lycopersicum esculentum Mill.). Revista Amazónica Ciencia y Tecnología 6:9-20.

Gutiérrez Brower, J., A. Romero, and A. K. Bulak. 2013. Selección de cepas de Trichoderma spp. de suelos lateríticos (Misiones, Argentina) según su capacidad antagonista in vitro y multiplicativa en cultivo bifásico y evaluación de su tolerancia a fungicidas químicos de uso habitual en cultivos de la zona. Revista Argentina de Microbiología 45:219.

Grijalba, P. E., G. A. Devitto, E. R. Wright, M. V. López, and O. S. Delfino. 1992. Eficiencia antagónica “in vitro" y en invernáculo de Trichoderma koningii sobre Colletotrichum dematium var. truncata. Revista Facultad de Agronomía 13: 157-162.

Haller, A. 1768. Historia stirpium indigenarum Helvetiae inchoata 1:250.

Hapon, M., P. Pizzuolo, G. Lucero, and J. Boiteux. 2010. Actividad biológica de metabolitos extracelulares producidos por aislados de Trichoderma spp. sobre Botrytis cinerea. Revista Argentina de Microbiología 42:213.

Harries, E., G. E. Mercado Cárdena, and L. Berruezo. 2017. Control biológico de Rhizoctonia solani por Trichoderma sp. aislados de suelos tabacaleros de Salta. Resumen $4^{\circ}$ Congreso Argentino de Fitopatología. Asociación Argentina de Fitopatólogos, Mendoza, Argentina. Pp. 361. URL: https://tinyurl.com/scqx3xp.

Howel, C. R. 2003. Mechanisms employed by Trichoderma Species in the biological control of plant diseases: The history and evolution of current concepts. Plant Disease 87:1-10. https://doi.org/10.1094/PDIS.2003.87.1.4.

Infante, D., B. Martínez, N. González, and Y. Reyes. 2009. Mecanismos de acción de Trichoderma frente a hongos fitopatógenos. Revista de Protección Vegetal 24:14-21.

Jaldo, A., and M. Delia. 2017. Un análisis de la producción y comercio internacional de cebolla: situación y perspectivas de la cadena de valor en Argentina. URL: http://hdl.handle.net/20.500.12123/1819.

Katinas L., D. G. Gutiérrez, M. Grossi, and J. Crisci. 2007. Panorama de la familia Asteraceae (=Compositae) en la República Argentina. Boletín de la Sociedad Argentina de Botánica 42: 113-129.

Kogan, M. 1998. Integrated pest management: historical perspectives and contemporary developments. Annual Review of Entomology 43:243-270. https://doi.org/10.1146/annurev.ento.43.1.243.

Linneo C. 1753. Species Plantarum Sections I-III. Pp. 127.

Lolas, M., and C. Sandoval. 2013. Control biológico de enfermedades fungosas en diferentes hortalizas. En J. R. Montealegre y L. M. Pérez (eds.). Control biológico de enfermedades de las plantas en Chile. Universidad de Chile, Santiago, Chile. Pp. 79-85.

Lucero, G., P. Pizzuolo, and M. Hapon. 2008. Acción antagónica "in vitro" de Trichoderma sp. sobre Botrytis cinerea, responsable de la podredumbre gris de la vid. Revista Enología 1:4.

Lucero, G., P. Boiteux, A. Alvarado, M. Díaz, and M. Hapon. 2014. Biocontrol de Phytophthora capsici por antibiosis o 
competición con tres aislados de Trichoderma sp. Resúmenes del $3^{\circ}$ Congreso Argentino de Fitopatología. Asociación Argentina de Fitopatólogos, San Miguel de Tucumán, Argentina. Pp. 386. URL: https://tinyurl.com/w4ozbhl.

Maumary, R., E. Zaiser, and M. Cracogna. 2017. Biocontrol de Macrophomina phaseolina por cepas de Trichoderma sp. aisladas de soja del norte de Santa Fe. Resumen $4^{\circ}$ Congreso Argentino de Fitopatología. Asociación Argentina de Fitopatólogos, Mendoza, Argentina. Pp. 385. URL: https://tinyurl.com/scqx3xp.

Mazza de Gaiad, S. M., S. A. Gutiérrez de Arriola and M. A. Cúndom. 2003. Selection of Trichoderma spp. isolates against Rhizoctonia solani. Span. Journal of Agricultural Research 4:79-82. https://doi.org/10.5424/sjar/2003014-53.

Menéndez, A. B., and A. Godeas. 1998. Biological control of Sclerotinia sclerotiorum attacking soybean plants. Degradation of the cell walls of this pathogen by Trichoderma harzianum (BAFC 742). Mycopathologia 142:153-60. https:/ /doi.org/ 10.1023/A:1006910707804.

Miralles, D., and F. González. 2010. El trigo en Argentina: Perspectivas ecofisiológicas del pasado, presente y futuro para aumentar el rendimiento. XVII Congreso de AAPRESID, La era del Ecoprogreso. Rosario, Santa fe, Argentina.

Mónaco, C., A. Pérelló, and M. C. Rollán. 1994. Ensayos in vitro del comportamiento antagónico de Trichoderma spp. frente a especies patógenas de la zona hortícola de La Plata, Argentina. Microbiología SEM 10:423-428.

Monroy, C., A. Cortés, D. Sicard, and H. Groot de Restrepo. 2005. Cytotoxicity and genotoxicity of human cells exposed in vitro to glyphosate. Biomédica 25:335-45. https://doi.org/10.7705/biomedica.v25i3.1358.

Montagna, M., and P. Collins. 2004. Efecto de un formulado comercial del herbicida glifosato sobre el cangrejo Trichodactylus borellianus (Crustacea, Decapoda: Braquiura). Revista de la Facultad de Bioquímica y Ciencias Biológicas 8:227-234. https://doi.org/10.14409/fabicib.v8i1.750.

Mukherjee, P. K., B. A. Horwitz, A. Herrera Estrella, M. Schmoll, and C. M. Kenerley. 2013. Trichoderma Research in the Genome Era. Annual Review of Phytopathology 51:105-29. https://doi.org/10.1146/annurev-phyto-082712102353.

Murillo, B. G., E. E. Guerrero, and S. R. Zapata. 2016. Manejo ecológico en frutilla aplicando Trichoderma sp. como promotor de crecimiento y controlador biológico de Botrytis cinérea. Avances en Energías Renovables y Medio Ambiente 20:09.37-09.44.

Nees, C. D. 1817. System der Pilze und Schwämme. Pp. 1-334.

Oddino, C. F. Giordano, J. Paredes, L. Cazón, J. Giuggia, and A. Rago. 2018. Efecto de nuevos fungicidas en el control de viruela del maní y el rendimiento del cultivo. Ab Intus - Facultad de Agronomía y Veterinaria 1: 9-17.

Paganelli, A., V. Gnazzo, H. Acosta, S. L. López, and A. E. Carrasco. 2010. Glyphosate based herbicides produce teratogenic effects on vertebrates by impairing retinoic acid signaling. Chemical Research in Toxicology 23:1586-1595. https://doi.org/10.1021/tx1001749.

Pearson, R., and A. Goheen. 1996. Plagas y Enfermedades de la Vid. The American Phytopathological Society. Ediciones Mundi-Prensa. Pp. 91.

Perello, A. E., C. I. Monaco, M. R. Simon, M. Sisterna, and G. Dal Belloa. 2003. Biocontrol efficacy of Trichoderma isolates for tan spot of wheat in Argentina. Crop Protection 22:1099-1106. https://doi.org/10.1016/S0261-2194(03)00143-1.

Perello, A. E., M. V. Moreno, C. Mónaco, M. R. Simón, and C. Cordo. 2009. Biological control of Septoria tritici bloth on wheat by Trichoderma spp. under field conditions in Argentina. BioControl 54:113-122. https://doi.org/10.1007/ s10526-008-9159-8.

Perniola, O. S., S. Staltari, S. E. Chorzempa, M. M. Astiz Gassó, and M. C. Molina. 2014. Control biológico de Fusarium graminearum: utilización de Trichoderma spp. y biofumigación con parte aérea de Brassica juncea. Revista de la Facultad de Ciencias Agrarias 46:45-56.

Persoon, C. H. 1794. Disposita methodica fungorum. Neues Magazin für die Botanik 1:81-128.

Pimentel, D., L. McLaughlin, A. Zepp, B. Lakitan, T. Kraus, P. Kleinman, F. Vancini, W. John Roach, E. Graap, W. S. Keeton, and G. Selig. 1991. Environmental and economic effects of reducing pesticide use. Bioscience 41:402-409. https://doi.org/10.2307/1311747.

Pineda Insuasti, J. A., E. N. Benavides Sotelo, A. S. Duarte Trujillo, C. A. Burgos Rada, C. P. Soto Arroyave, C. A. Pineda Soto, C. A. Fierro Ramos, F. J. Mora Muñoz, E. Sulay, and S. E. Álvarez Ramos. 2017. Producción de biopreparados de Trichoderma spp: una revisión. ICIDCA. Sobre los Derivados de la Caña de Azúcar 51:47-52.

Pringsheim, N. 1858. Beiträge zur Morphologie and Systematik der Algen. 2. Die Saprolegnieen. Jahrbücher für Wissenschaftliche Botanik 1:284-306.

Rahman, A., M. F. Begum, M. Rahman, M. A. Bari, G. N. Ilias, and M. F. Alam. 2011. Isolation and identification of Trichoderma species from different habitats and their use for bioconversion of solid waste. Turkish Journal of Biology 35:183-194.

Reca, L. G., and G. H. Parellada. 2001. La agricultura argentina a comienzos del milenio: Logros y desafíos. Desarrollo Económico. Pp. 707-737. https://doi.org/10.2307/3456001.

Reznikov, S., G. R. Vellicce, V. González, V. De Lisi, A. P. Castagnaro, and L. D. Ploper. 2016. Evaluation of chemical and biological seed treatments to control charcoal rot of soybean. Journal of General Plant Pathology 82:273-280. DOI: 10.1007/s10327-016-0669-4. https://doi.org/10.1007/s10327-016-0669-4.

Rifai, M. A. 1969. A revision of the genus Trichoderma. Mycological Papers 116:1-56.

Rivera, M., and E. Wright. 2014. Control biológico de enfermedades de plantas en Argentina. En B. Wagner, M. Rivera, P. Mondino, J. Montealegre and Colmenárez (eds.). Control biológico de enfermedades de plantas en América Latina y el Caribe. Pp. 9-81.

Rodríguez, A. V., G. G. Cordes, F. B. Godoy, and J. R. Pasquini. 2017. Evaluación de aislamientos de Trichoderma spp. contra Sclerotium rolfsii y Sclerotinia minor bajo condiciones in vitro. Resumen $4{ }^{\circ}$ Congreso Argentino de Fitopatologia. Asociación Argentina de Fitopatólogos, Mendoza, Argentina. Pp. 318. URL: https://tinyurl.com/scqx3xp.

Rojas Molina F., and Williner V. 2013. First record of the non-indigenous mussel Limnoperna fortunei (Bivalvia, Mytilidae) as an epibiont of the crab Trichodactylus borellianus (Decapoda, Trichodactylidae). Crustaceana 86:682-692. https: //doi.org/10.1163/15685403-00003183.

Rojo, G., M. Reynoso, M. Ferez, S. Chulze, and A.Torres. 2006. Biological control by Trichoderma species of Fusarium solani causing peanut brown root rot under field conditions. Crop Protection 26:549-555. https://doi.org/10.1016 
i.cropro.2006.05.006

Rojo, G., M. Ferez, M. Reynoso, A. Torres, and S. Chulze. 2007. Effect of Trichoderma species on growth of F. proliferatiom and production of fumonisins, fusaproliferin and beauvericin. Mycotoxin Research 23:173-179. https://doi.org/ $10.1007 /$ BF02946044.

Saccardo, P. A. 1880. Conspectus generum fungorum Italiae inferiorum nempe ad Sphaeropsideas, Melanconieas et Hyphomyceteas pertinentium systemate sporologico dispositorum. Michelia 2:1-38.

Saccardo, P. A. 1911. Notae mycologicae. Series XIII. Annales Mycologici 9:249-257. Samuels, G. J., E. Lieckfeldt, and H. I. Nirenberg. 1999. Trichoderma asperellum, a new species with warted conidia, and redescription of $T$. viride. Sydowia 51:71-88.

Samuels, G. J., S. Dodd, B. Lu, O. Petrini, H. J. Schroers, and I. Druzhinina. 2006. The Trichoderma koningii aggregate species. Studies in Mycology 56:67-133. https://doi.org/10.3114/sim.2006.56.03.

Sánchez, A. D., V. Barrera, G. E. Reybet, and M. C. Sosa. 2015. Biocontrol con Trichoderma spp. de Fusarium oxysporum causal del "mal de almácigos" en pre y post emergencia en cebolla. Revista de la Facultad de Agronomía, La Plata 114:61-70.

Sánchez, S., and M. M. Gassó. 2017. Control biológico de R. solani en Lycopersicum esculentum con Trichoderma sp. en invernáculo. Resumen $4^{\circ}$ Congreso Argentino de Fitopatología. Asociación Argentina de Fitopatólogos, Mendoza, Argentina. Pp. 308. URL: https://tinyurl.com/scqx3xp.

Schliserman, P., and S. M. Ovruski, S. M. 2004. Incidencia de moscas de la fruta de importancia económica sobre Citrus aurantium (Rutaceae) en Tucumán, Argentina. Manejo Integrado de Plagas y Agroecología Costa Rica 72:52-61.

Schultz, H. 1936. Vergleichende Untersuchungen zur Ökologie, Morphologie und Systematik des "Vermehrungspilzes". Arbeiten der Biologischen Reichsanstalt für Land- und Forstwirtschaft 22:1-42.

Sharma, P., M. Sharma, M. Raja and V. Shanmugam. 2014. Status of Trichoderma research in India: A review. Indian Phytopathology 67:1-19.

Shoemaker, R. A. 1959. Nomenclature of Drechslera and Bipolaris, grass parasites segregated from 'Helminosporium'. Canadian Journal of Botany 37:879-887. https://doi.org/10.1139/b59-073.

Shoemaker, R. A. 1962. Drechslera Ito. Canadian Journal of Botany 40:809-836. https:/ /doi.org/10.1139/b62-075.

Singh, D., and S. K. Mathur. 1974. S. rolfsii in seeds of bean from Uganda. Seed Science and Technology 2:481-483.

Stepin-Sytnik, E. V., M. C. Garay-Villalba, M. Maidana-Ojeda, and C. Cabral-Antúnez 2018. Insectos depredadores y parasitoides asociados al cultivo de soya (Glycine max L. Merrill, 1917), en el distrito Arquitecto Tomás Romero Pereira, Itapúa, Paraguay. Intropica 13:122-129. https:/ / doi.org/10.21676/23897864.2611.

Stocco, M. C., C. I. Monaco, C. Abramoff, G. Lampugnani, G. Salerno, N. Kripelz, C. A. Cordo, and V. F. Consolo. 2016. Selection and characterization of Argentine isolates of Trichoderma harzianum for effective biocontrol of Septoria leaf blotch of wheat. World Journal of Microbiology and Biotechnology 32:49. https://doi.org/10.1007/s11274-0151989-9.

Traglia, C., M. Vidal, J. Gortari, V. Rosenfeld, and A. Oviedo. 2018. Concentración económica en cadenas agroindustriales de Misiones, Argentina: yerba mate, té y mandioca. SaberEs 10:19-36.

Valetti, L., L. I. Cazón, and S. Pastor. 2017. Potencial de Trichoderma spp. como agente biocontrolador de cancrosis papirácea del manzano causada por Valsa ceratosperma. Resumen $4^{\circ}$ Congreso Argentino de Fitopatología. Asociación Argentina de Fitopatólogos, Mendoza, Argentina. Pp. 329. URL: https://tinyurl.com/scqx3xp.

Vázquez, M. A., E. Maturano, A. Etchegoyen, F. S. Difilippo, and B. Maclean. 2017. Asociación entre cáncer y exposición ambiental a glifosato. International Journal of Clinical Medicine 8:73-85. https://doi.org/10.4236/ijcm.2017.82007.

Vereschuk, M. L., A. C. López, A. S. Chelaliche, A. E. Alvarenga, E. Schegg E, G. A. Netter, M. F. Luna, and L. L. Villalba. 2017. Actividad antagónica de aislados de Trichoderma sp. endófitos de yerba mate (Ilex paraguariensis). Resumen $4^{\circ}$ Congreso Argentino de Fitopatología. Asociación Argentina de Fitopatólogos, Mendoza, Argentina. Pp. 300. URL: https://tinyurl.com/scqx3xp.

Viera, W., M. Noboa, J. Bermeo, F. Báez, and T. Jackson. 2018. Parámetros de calidad de cuatro tipos de formulaciones a base de Trichoderma asperellum y Purpuricillium lilacinum. Enfoque UTE 9:145-153. https://doi.org/10.29019/ enfoqueute.v9n4.348.

Vinale, F., K. Sivasithamparam, E. L. Ghisalberti, R. Marra, S. L. Woo, and M. Lorito. 2008. Trichoderma-plant-pathogen interactions. Soil Biology and Biochemistry 40:1-10. https:/ / doi.org/10.1016/j.soilbio.2007.07.002.

Vogrig, J. A., S. Sarrailhé, M. A. López Amaya, O. S. Correa, and M. S. Montecchia. 2017. Capacidad antagónica de cepas de Trichoderma nativas de suelos del noroeste Argentino contra Macrophomina phaseolina. Resumen $4^{\circ}$ Congreso Argentino de Fitopatología. Asociación Argentina de Fitopatólogos, Mendoza, Argentina. Pp. 331 URL: https:// tinyurl.com/scqx3xp.

Walsh, L. P., C. McCormick, C. Martin, and D. M. Stocco. 2000. Roundup inhibits steroidogenesis by disrupting steroidogenic acute regulatory (StAR) protein expression. Environmental Health Perspectives 108:769-776. https: //doi.org/10.1289/ehp.00108769.

Weindling, R. 1934. Studies on a lethal principle effective in the parasitic action of Trichoderma lignorum on Rhizoctonia solani and other soil fungi. Phytopathology 24:1153-79.

Wells, H. D., D. K. Bell, and C. A. Jaworski. 1972. Efficacy of Trichoderma harzianum as a biological control for Sclerotium rolfsii. Phytopathology 62:442-47. https://doi.org/10.1094/Phyto-62-442.

Wollenweber, H. W. 1930. Fusaria Autographice Delineata 3:660-1100.

Woo, S. L., M. Ruocco, F. Vinale, M. Nigro, R. Marra, N. Lombardi, A. Pascale, S. Lanzuise, G. Manganiello, and M. Lorito. 2014. Trichoderma- based products and their widespread use in agriculture. The Open Mycology Journal 8: 71-126. https://doi.org/10.2174/1874437001408010071.

Wright, E. R., R. Zapata, O. S. Delfino, M. V. López, and M. Senlle. 1988. Eficiencia in vitro de antagonistas de S. sclerotiorum y S. minor. Revista Facultad de Agronomía 9:119-116.

Zapata, R. L., S. R. Spivak, O. S. Filippini de Delfino, and M. C. Fabrizio. 1997. Control de la podredumbre de la endivia (Cichorium intybus L. var. foliosum) producida por Sclerotinia sclerotiorum mediante la aplicación de Trichoderma harzianum. Revista Facultad de Agronomía 17:151-155. 